\title{
El enfoque de marco lógico y los proyectos de desarrollo: el caso de Fondoempleo durante los años 2006 a 2010 en Perú
}

Sección ESTUDIOS

RECIBIDO: 08/08/2021

APROBADO: $28 / 08 / 2020$

PUBLICADO ONLINE: 30/12/2021

\author{
Ernesto Sueiro Cabredo \\ Universidad Nacional Mayor de San Marcos \\ esueiro67@gmail.com \\ https:/0rcid.org/0000-0002-2770-0707
}

\section{RESUMEN}

Esta investigación busca describir y analizar las disparidades encontradas en la definición de Fin y Propósito, así como los Indicadores de Impacto y Efecto a través del uso del enfoque de marco lógico en los concursos de Fondoempleo de los años 2006 a 2009. Para ello, se realizó una revisión documental de los manuales como de los proyectos ganadores de los concursos de la organización; además, se realizaron entrevistas a evaluadores y ejecutores. Esto nos permitió conocer las diversas connotaciones contradictorias tanto en la definición y aplicación práctica, así como la participación en la reducción de la pobreza en los proyectos sociales; asimismo, se analizaron ejemplos de proyectos ganadores.

PALABRAS CLAVE: Marco lógico, proyectos sociales, desarrollo, pobreza.

\section{The logical framework approach and development projects: The case of fondoempleo during the years 2006 to 2010 in Peru}

\section{ABSTRACT}

This research seeks to describe and analyze the disparities found in the definition of Aim and Purpose, as well as the Impact and Effect Indicators through the use of the Logical Framework Approach in the Employment Fund contests from 2006 to 2009. To do so, made a documentary review of the manuals and of the winning projects of the organisation's contests; In addition, evaluators and executors were interviewed. This allowed us to know the various contradictory connotations both in the definition and practical application, as well as the participation in poverty reduction in social projects; Examples of winning projects were also analyzed.

KEYWORDS: Logical framework, social proyects, development, poverty.

(c) Los autores. Este artículo es publicado por la Revista de Sociología de la Facultad de Ciencias Sociales, Universidad Nacional Mayor de San Marcos. Este es un artículo de acceso abierto, distribuido bajo los términos de la licencia Creative Commons Atribución 4.0 Internacional (CC BY 4.0) [https://creativecommons.org/licenses/by/4.0/deed.es] que permite el uso, distribución y reproducción en cualquier medio, siempre que la obra original sea debidamente citada de su fuente original. 


\section{Introducción}

E n los últimos años se ha planteado desde entidades multinacionales como el Banco Mundial, el Banco Interamericano de Desarrollo, la Organización de las Naciones Unidas, entre otras, programas y proyectos sociales que han sido diseñados a partir de la aplicación del método del marco lógico. La importancia de considerar los programas y proyectos de carácter social y vinculados al desarrollo se debe a su relación con la búsqueda de mejorar la realidad y brindar soluciones a los problemas identificados, en tanto que "distribuyen bienes o servicios (productos), para satisfacer las necesidades de aquellos grupos que no poseen los recursos para solventarlas autónomamente" (Cohen y Martínez, 2004, p. 22). A partir de esta intervención, se espera lograr un cambio o transformación social, enfatizando en la necesidad de que sea un proceso participativo $y$, en consecuencia, sostenible (Baca y Herrera, 2016).

En este sentido, el método del marco lógico se considera como una herramienta que permita la correcta ejecución de programas y proyectos sociales, dado que facilita un formato unificado de planificación en diversos aspectos. Por ello, siguiendo a Gonzales y Zanfrillo (2006), el marco lógico se convierte en un instrumento efectivo para el desarrollo de proyectos con enfoque social. Sin embargo, a pesar de su importancia, se encuentra que el método del marco lógico, en la realidad, no logra plasmar en los programas y proyectos sociales sus objetivos y beneficios, simplificando y reduciendo la complejidad de la problemática social (Rodríguez, 2008) y, en cambio, logrando establecer una estructura rígida.

Por ello, investigaciones realizadas encontraron fallas vinculadas a su implementación en cuanto a contenido y definición de indicadores (Bueno, 2014), o una lógica débil entre la población objetivo y lo obtenido a partir de la aplicación del método dificultando así el logro de metas (Sánchez, 2011). Así, a pesar de lo fundamental que resulta la correcta aplicación del marco lógico en los proyectos sociales de desarrollo, se encuentra la existencia de disparidades entre lo propuesto y lo ejecutado desde sus componentes como los objetivos e indicadores.

Considerando esta problemática, mediante esta investigación se busca conocer cómo inciden las diferentes nociones de objetivos e indicadores del método del marco lógico en los proyectos sociales de desarrollo de Fondoempleo, tomando en cuenta la información de manuales y guías contrastadas con los aspectos de la realidad concreta. Este objetivo se justifica en tanto que, actualmente, no se encuentra un consenso en el caso peruano para la definición de conceptos y metodología que 
permita analizar programas y proyectos sociales, encontrándose así una necesidad de conocer las diferencias y lograr consensos a partir de estas alternativas.

\section{Antecedentes y aspectos teóricos}

Desde las entidades de cooperación y desarrollo como el Banco Mundial, el Banco Interamericano de Desarrollo, GTZ o el Fondo Internacional de Desarrollo Agropecuario, en general, si bien se han encontrado similitudes en la estructura de la matriz de marco lógico como, por ejemplo, su composición de cuatro filas y cuatro columnas, en el contenido se evidencian diferencias de denominación y vacíos en cuanto a lo que debería abordar cada uno de sus componentes y niveles. Similar situación se encuentra en instituciones como Cepal o las vinculadas a gobiernos como lo presentado por documentos de la Unión Europea o el Ministerio de Economía y Finanzas para el caso peruano.

A partir de esta situación, se considera importante conocer el impacto del enfoque de marco lógico en los programas y proyectos sociales, así como en la administración pública. Así, el uso del marco lógico en las diferentes etapas de un proyecto es fundamental. En el diagnóstico, como primer paso, establece un análisis de problemáticas, objetivos, actores y propuestas de alternativas de solución, encontrándose por eso necesario que el proceso sea contextualizado y participativo, tal como se señala a continuación:

El marco lógico tiene la intención de lograr una definición realista y clara de los objetivos en una perspectiva a largo plazo, mejorar la comunicación y la organización de contrapartes, a través de la planificación conjunta, utilizando documentos y definiciones claras, y establecer los indicadores para la evaluación. (Baca y Herrera, 2016, p. 212)

En cuanto a la formulación, esta etapa nos ayuda a la definición de objetivos y logros de metas, articulando lo requerido para la solución de problemas y el impacto esperado. Aquí, desde el marco lógico, la matriz permite la organización y síntesis de la información obtenida.

La matriz es un marco lógico que permite organizar y resumir la información relevante de cada alternativa (objetivos de impacto, objetivos de producto, metas, fuentes de verificación y supuestos) y realizar comparaciones entre ellas. (Cohen y Martínez, 2004, p. 39) 
En la etapa de evaluación, ya sea ex ante o ex post, se resaltan los términos de eficacia y eficiencia $y$, aunque es un proceso fundamental dado que permite el logro de los objetivos del proyecto, no suele realizarse de manera correcta o con metodologías adecuadas, tal como se menciona a continuación:

Hay que aumentar la racionalidad con que se utilizan los recursos destinados a lo social. Ello sólo será posible midiendo la eficiencia con la cual son utilizados y comprobando la eficacia con que se alcanzan los objetivos de los programas. Para ello se requiere evaluarlos. (Cohen y Franco, 1988, p. 16)

Sin embargo, no se debe dejar de lado los aspectos sociales vinculados a la eficacia y la eficiencia relacionados a la equidad, en donde, a pesar de que se consideran necesarios para la productividad, también conllevan aspectos de desigualdad. Asimismo, según Perrow (1992), no deben ser considerados de manera reduccionista a cuestiones burocráticas o de crecimiento, sino también se debe considerar el uso del trabajo, capital y recursos naturales.

Tomando en cuenta esta relación del marco lógico en las etapas de un proyecto social, se encuentra que, si bien frecuentemente se utiliza el marco lógico en ellas por su sencillez y dinamismo (Loor, Loor y Bravo, 2012), se presentan dificultades en esa aplicación al no lograr plasmar todos los aspectos y la complejidad de las problemáticas sociales encontradas en la realidad, generando así un enfoque utilitarista (Rodríguez, 2008).

En cuanto a la aplicación del marco lógico en la administración pública, al igual que en los proyectos sociales, se espera que, dadas sus características, permita una correcta identificación del problema, propuestas de solución y con enfoque participativo (Sánchez, 2007). Sin embargo, en este caso, el uso del marco lógico puede convertirse en una herramienta reguladora, en tanto que, un directivo no solo está presente en la etapa de diseño, sino también en la coordinación (Culebro y Barragán, 2014).

A continuación, reconociendo la relación del marco lógico con temas de desarrollo y, en consecuencia, la lucha por superar desigualdades es importante mencionar que, según Franco (1996), se identifican tres modelos de desarrollo vinculado a la política social: primero un crecimiento hacia afuera con predominancia de exportación de materias primas; segundo, la sustitución de importaciones en donde el Estado asume nuevas responsabilidades; y tercero, la competitividad y competencia internacional.

A partir de esta situación latinoamericana, en el Perú se desarrollaron diversos programas y proyectos sociales que buscaban superar las brechas y desigualdades, 
como es el caso del Programa de Capacitación Laboral Juvenil (ProJoven) vinculada a la mejora laboral de los jóvenes en situación de pobreza y pobreza extrema, de las mujeres, reducir la inactividad, entre otros (Saavedra y Chacaltana, 2000). En cuanto al rubro de la alimentación, Alcázar (2007) resalta lo realizado por los Comedores populares, Qali Warma y Wawa Wasi. Sin embargo, en este caso, los resultados no fueron los esperados por problemas de enfoque y diseño. Vinculado a la pobreza, según Sánchez y Rodríguez (2016) el Programa Nacional de Apoyo Directo a los más Pobres-Juntos, que busca lograr el acceso a servicios de salud y educación en hogares de bajas condiciones económicas, ha logrado mejoras en su intervención.

Ahora, desde una perspectiva teórica vinculada a la sociología de las organizaciones, se encuentra que, según González-Anleo (1999), resaltan tres periodos: primero, una perspectiva clásica de modelo weberiano que enfatiza sobre la racionalidad y la eficacia en entornos burocráticos, como también de un modelo que enfatiza en el trabajo despersonalizado y rígido. Un segundo periodo, por el contrario, tiene un enfoque humanista que toma en cuenta las necesidades de los trabajadores. El tercer periodo es neorracionalista que busca integrar los periodos anteriores, entendiendo a la organización como un sistema abierto. A partir de estos periodos, se brinda un concepto de organización, entendida como:

Una asociación permanente de individuos que con la intención de conseguir fines específicos coordinan sus actividades mediante la formalización y reglamentación de las mismas y mediante el desempeño de roles específicos que se articulan en estructuras diferenciadas y jerarquizadas. (González-Anleo, 1999, p. 42)

Asimismo, desde una perspectiva neoinstitucionalista, se encuentran cambios que viabilizan el surgimiento del campo organizacional, lo cual posibilita que el análisis no solo se limite a las organizaciones, sino que también considere a los actores:

Por campo organizacional nos referimos a aquellas organizaciones que, en conjunto, constituyen un área reconocida de la vida institucional: los proveedores principales, los consumidores de recursos y productos, las agencias reguladoras y otras organizaciones que dan servicios o productos similares. (Powell y Dimaggio, 1999, p. 106)

Al respecto se puede señalar que las organizaciones se encuentran cada vez más en una situación de homogeneidad y de conocimiento no solo de sus aspectos internos, sino de consideraciones externas. Así, el término isomorfismo, según Powell y Dimaggio (1999) surge como el que mejor describe esta situación “El isomorfismo 
es un proceso limitador que obliga a una unidad en una población a parecerse a otras unidades que enfrentan las mismas condiciones ambientales" (p.108).

De igual manera, la doble contingencia, entendida como el hecho de que se es gente actuante y objeto de actuación dependiendo de factores internos y externos, facilita decisiones organizacionales anticipadas según el conocimiento que se tenga del otro, tal como se menciona a continuación: "La doble contingencia supone que cada uno de los actores en una situación interactiva es, a un tiempo, agente de acciones para sí y para otros; pero es también objeto de acciones para sí y para otros" (Izuzquiza, 2008, p.244).

Como se observa, en el planteamiento del problema, revisión de antecedentes y revisión teórica, si bien se reconoce la importancia de la incorporación del enfoque de marco lógico tanto en proyectos sociales como en la administración pública relacionados al desarrollo y superación de la pobreza, su implementación se ha caracterizado por presentar inconsistencias que no permiten aprovechar todos sus beneficios.

\section{Metodología}

Esta investigación se realizó desde un enfoque cualitativo dado que buscamos comprender los aspectos de la realidad social que consideran los discursos y percepciones de los actores relacionados con el diseño y la evaluación de los proyectos sociales de desarrollo vinculados a Fondoempleo. Tal como señala Mejía (2004), la investigación cualitativa toma en cuenta discursos que facilitan la comprensión de la vida social. Ello se relaciona con nuestro planteamiento epistemológico que considera central al actor en este análisis.

La investigación abarca el periodo de 2006 a 2010 y considera como población a los proyectos sociales de desarrollo de temática agropecuaria realizadas en zona con alto índice de pobreza. A partir de ello, se define la muestra desde un estudio de caso: los proyectos ganadores de los concursos realizados por Fondoempleo. Los criterios por los cuales se seleccionó a esta organización es que se considera representativa de las entidades que proporcionan fondos concursables para proyectos de desarrollo social públicos y privados; asimismo, no ha suspendido la realización de sus concursos; y, por último, se cuenta con disponibilidad de acceso a la información lo cual viabiliza la investigación.

Tomando en cuenta el periodo de tiempo planteado para esta investigación, se han considerado documentos y actores representativos: 
- Manualeseinstructivos parala participación en los concursos deFondoempleo.

- Proyectos ganadores que fueron ejecutados.

- Dos ejecutores de proyectos con conocimiento del funcionamiento de la organización.

- Dos evaluadores de proyectos con conocimiento del funcionamiento de la organización.

Para la recolección de información, considerando los aspectos metodológicos y epistemológicos planteados, se aplicaron técnicas como: la entrevista, que nos permite una recolección primaria dirigida a los ejecutores y evaluadores, y el análisis documental, para conocer información secundaria de los manuales, instructivos y documentos producto de la ejecución de los proyectos ganadores.

La información obtenida fue analizada según cada técnica e instrumento: Las entrevistas fueron grabadas previa autorización del o la entrevistado/a y luego transcritas, permitiendo ello su categorización y análisis. En cuanto al análisis de documentos, estos fueron revisados y categorizados en un archivo Excel, seleccionando la información más relevante.

\section{Las diversas connotaciones del enfoque de marco lógico en el caso de Fondoempleo}

\section{Desde su estructura y sus elementos}

En la aplicación del enfoque de marco lógico y su matriz correspondiente, se encuentra que, a pesar de que los beneficios de este enfoque que pueden significar resultados positivos, muchos proyectos o programas no presentan coherencia entre lo diagnosticado o lo plasmado en la matriz; en consecuencia, los indicadores planteados no son pertinentes y claros (Bueno, 2014).

En cuanto a la coherencia sobre el uso de la matriz de marco lógico en los manuales de formulación de proyectos del Fondoempleo, en general, tanto los evaluadores como los ejecutores señalan que existe coherencia, aunque también consideran que no necesariamente se cumple siempre. Así, según el evaluador 1 entrevistado:

En términos de material de capacitación y según lo explicado en los talleres que el fondo realiza para quienes presentan sus propuestas, sí hay una coherencia básica 
respecto a cómo hacer los proyectos. Esta no siempre es detallada o desagregada. Sin embargo, al leer los proyectos que son adjudicados no siempre hay coherencia. He sido testigo de cómo algunos proyectos fueron "ajustados" por el Fondo para ser más coherentes, los resultados no siempre fueron favorables. (Evaluador 1)

La coherencia en los proyectos ha ido mejorando, pero donde se encuentra más deficiencia es en los indicadores, dado que estos son inadecuados en comparación con los recursos disponibles. En palabras de evaluador 2 entrevistado:

En cada una de las rondas, los documentos guía han ido mejorando para tratar de lograr esa coherencia. Sin embargo, en los proyectos que evaluamos no se logra plantear adecuadamente los objetivos, los IVOs y la lógica vertical, ya que la relación entre componentes y propósito muchas veces no es tal. El manejo de indicadores y la posibilidad de lograr con los recursos el propósito y la contribución al fin planteada, no resultaba adecuado, ya que se plantean metas e indicadores inapropiados para la cantidad de recursos y tiempo de ejecución del proyecto. Quizás algunos buenos ejemplos (de distintos tipos de proyectos) deberían estar a disposición de los participantes. (Evaluador 2)

Es importante mencionar que, respecto a la coherencia, la respuesta de los evaluadores, a diferencia de los ejecutores, es más profunda y detallada. Esto puede explicarse por los diferentes roles de cada uno: los evaluadores miran y opinan desde su rol, lo que su experiencia les ha demostrado en más de un proyecto; mientras que los ejecutores, desde su rol, aceptan sin ningún cuestionamiento pues han logrado obtener un financiamiento a partir de lo que diseñaron.

Además, si bien los manuales y documentos de Fondoempleo señalan que son 16 los espacios con los que debe contar una matriz de marco lógico, en el cual los elementos de la columna izquierda (fin, propósito, resultados y actividades) incluyen necesariamente información correspondiente a las otras tres columnas (indicadores, medios de verificación y supuestos), se observa que desde Fondoempleo no exigen que se cumpla esta condición en los proyectos ganadores. Así, se encuentran proyectos que presentan una finalidad clara, pero sus indicadores, medios de verificación y supuestos no necesariamente se encuentran acordes o pertinentes con aquella información.

Las inconsistencias también se presentan en los objetivos redactados para el fin, a pesar de que el planteamiento correcto de objetivos es fundamental para capturar la complejidad de la realidad social (Bueno, 2014; Rodríguez, 2008). Sin embargo, uno de los problemas más evidentes al plantear objetivos es la inclusión de la reducción de la pobreza a pesar de que esto no es pertinente ni lograble en un proyecto. Considerando lo señalado por evaluador 1: 
Es un anhelo de todo proyecto de desarrollo promover grandes cambios en sus beneficiarios. La meta de pobreza es quizá el cambio más grande que se pueda buscar, pero se debe entender la lógica y alcance de los proyectos, para poder entender que los proyectos son solo procesos. Bajo este esquema, superar la pobreza va mucho más allá de un proyecto. Creo que esta situación salió de algunas charlas de capacitación, hasta donde me parece, está ligado a que el fondo usó como ejemplo la superación de pobreza como finalidad sobre la cual se aporta. Esto y el hecho que la presentación del fondo reseña que busca ayudar en la superación de la condición de pobreza de la zona habrían generado este error sistemático. (Evaluador 1)

Así, se encuentra que en los proyectos se busca contribuir a la reducción de la pobreza como un deseo o voluntad, mas no de una posibilidad realista y posible en el marco de la ejecución de su proyecto, teniendo en cuenta también que, si desde los manuales se pide una mejora en los aspectos laborales, no solo ese sector de la sociedad reduce la pobreza, sino que esta es multidimensional.

Esta situación se relaciona con el impacto y sus indicadores, teniendo en cuenta que este se reconoce como el logro final a partir de la ejecución de un proyecto. Así, en palabras de ejecutor 1 y evaluador entrevistados:

El impacto del proyecto se mide en la capacidad de generar cambios significativos en la población objetivo, como consecuencia de los resultados logrados en el proceso de ejecución del proyecto. El impacto se constata luego de finalizado el proyecto, o por su posibilidad de réplica en otros grupos de población similares a los del proyecto. (Ejecutor 1)

El impacto se refiere al fin último al cual el proyecto contribuye con su ejecución. (Evaluador 2)

Observando ambas intervenciones, encontramos que el ejecutor 1 identifica al impacto con el propósito del proyecto (logro final de las acciones dentro de la ejecución del proyecto), mientras que el evaluador 2 identifica al impacto con la finalidad del proyecto (fin al cual contribuye el proyecto), por lo cual no hay un consenso.

A pesar de estas inconsistencias, en esta oportunidad los manuales de Fondoempleo establecen una convención en torno al impacto entendiéndolo como un indicador de finalidad, que evalúa el aporte al objetivo de un proyecto, y de efecto, al culminar la ejecución de un proyecto. 


\section{Desde su contribución a la reducción de la pobreza}

El vínculo del marco lógico con los proyectos de desarrollo y pobreza es necesario, sin embargo, se encontró que no necesariamente logran captar su complejidad ni, mediante sus indicadores, un impacto importante o que de manera directa logre su finalidad. Según el evaluador 2 entrevistado:

Muchos proyectos asumen que las mejoras que ellos brindan, como, por ejemplo, mayores ingresos o productividades u otros, no sólo permiten un mejor ingreso, sino que, asumen que este ingreso permite moverse de la situación de pobreza. Es importante distinguir que dadas las características del fondo esto no es fácilmente medible, pues la pobreza está relacionada con el gasto de una familia u hogar, y en ello, influye mucho más que tener más plata, es que esta plata vaya a subsanar, en orden de importancia, las falencias de alimentación, educación, salud, etc. Si bien, varios proyectos han propuesto este indicador, pocos incluyen su medición, pues lo consideran como "impacto indirecto" u "aporte a la finalidad", es decir, no tienen que rendir cuenta de ello, pero si lograr demostrar que coadyuvan a superar una situación crítica, en este caso, la pobreza. (Evaluador 1).

Entonces se evidencia la intención de incentivar a través de planteamiento de tales indicadores de propósito una reducción de la pobreza o mejora de la calidad de vida, sin embargo, estos no son factibles de lograr en el periodo de implementación de un proyecto, dado que rebasan el mismo. En palabras del ejecutor 1 entrevistado:

Reducción de pobreza, es un indicador cuya medición es compleja y el plazo para sus variaciones puede ser muy largo, rebasando la vida del proyecto. Sin embargo, constituye un criterio implícito, en el diseño del proyecto. La forma más simple de medirla es a través de los indicadores de incremento de ingresos y empleo en el proyecto, pero será necesario contrastar con los datos estadísticos del territorio más amplio (distrito, provincia) lo cual es muy difícil. (Ejecutor 1).

Por ello se considera más pertinente considerar que desde un proyecto no se puede reducir la pobreza, sino que debe ir más allá del fin y ser una meta que involucre diversos proyectos que apunten hacia diversos aspectos como la alimentación, la educación, la salud, la vivienda, etc. Así, un proyecto puede incidir, pero no lograr por sí mismo la reducción de la pobreza.

Así, en cuanto a lo que señalan los documentos de Fondoempleo, encontramos que, específicamente sobre el fin de un proyecto, este no necesariamente será logrado al finalizarlo porque implica un largo plazo más allá de su ejecución, es 
decir, contribuir a la solución de problemas de carácter nacional que logre impactar en cuestiones de desarrollo. Por ello, la ejecución del proyecto no necesariamente aporta al fin en su totalidad y los proyectos deben considerar como impacto solo las mejoras en aspectos laborales y promoción del empleo.

Al respecto, consideramos que el fin sí debe lograrse al finalizar un proyecto teniendo en cuenta que su propuesta debe ser realista y pertinente. Sin embargo, encontramos en los proyectos ganadores que el vínculo del fin con el desarrollo es una relación extrema no razonable de lo que puede aportar un proyecto en su ámbito de acción comparado con lo que supone debe aportar el desarrollo a nivel nacional. No se encuentra un aporte total dado que muchos proyectos incluso no consideran indicadores verificables objetivamente (IVO), además los proyectos ganadores no necesariamente cumplen con incluir impactos de mejora laboral, el cual es uno de los requisitos del Fondoempleo.

Por ello, a continuación, presentamos ejemplos seleccionados de los proyectos ganadores de Fondoempleo entre los años 2006 a 2009 que demuestran lo señalado en los párrafos previos.

En efecto, encontramos que el proyecto "Gestión agroecológica para la producción y comercialización en la microcuenca del río caudaloso" presenta como finalidad "contribuir a mejorar la calidad de vida de los pobladores de la microcuenca del río caudaloso"; sin embargo, no considera un indicador sobre cómo el proyecto lo logrará esa finalidad.

El proyecto "Mayor eficiencia competitiva para el posicionamiento comercial e incremento de empleo e ingresos, con las organizaciones de pequeños productores de banano y mango orgánicos del valle Alegre, Región S", presenta las siguientes características:

\section{Tabla 1}

Proyecto: Mayor eficiencia competitiva para el posicionamiento comercial e incremento de empleo e ingresos, con las organizaciones de pequeños productores de banano y mango orgánicos del valle Alegre, Región S.

\begin{tabular}{|l|l|l|l|}
\hline \multicolumn{1}{|c|}{ Objetivos } & \multicolumn{1}{|c|}{ Indicadores } & \multicolumn{1}{c|}{$\begin{array}{c}\text { Medios de } \\
\text { verificación }\end{array}$} & \multicolumn{1}{c|}{ Supuestos } \\
\hline $\begin{array}{l}\text { Finalidad: Contribuir al } \\
\text { proceso de desarrollo } \\
\text { económico local sostenible, } \\
\text { mejorando los ingresos de } \\
\text { los pequeños productores } \\
\text { agrarios de Valle Alegre en las } \\
\text { Provincias B y C - Región U. }\end{array}$ & $\begin{array}{l}\text { Mejoramiento del } \\
\text { índice de pobreza } \\
\text { de las provincias } \\
\text { By C. }\end{array}$ & $\begin{array}{l}\text { Mapa de pobreza } \\
\text { FONCODES }\end{array}$ & $\begin{array}{l}\text { Estabilidad social y } \\
\text { económica del país. }\end{array}$ \\
\hline
\end{tabular}

Fuente: Proyectos ganadores de concursos Fondoempleo, 2006-2009. 
Como se observa, lo que se considera como indicador no reúne los requisitos básicos como tal; no está claro en qué grado y en qué tiempo se mejorará el índice de pobreza. Además, se debe considerar que la pobreza es multidimensional, trascendiendo lo que un proyecto podría lograr.

El proyecto "Generación y consolidación de empleo sostenible en micro pro-

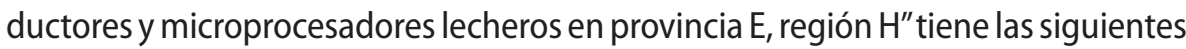
características:

\section{Tabla 2}

Proyecto: Generación y consolidación de empleo sostenible en micro productores y microprocesadores lecheros en provincia E, región $H$

\begin{tabular}{|c|c|c|c|}
\hline Objetivos & Indicadores & $\begin{array}{l}\text { Medios de } \\
\text { verificación }\end{array}$ & Supuestos \\
\hline $\begin{array}{l}\text { Finalidad: Contribuir a } \\
\text { reducir los índices de } \\
\text { pobreza extrema del sector } \\
\text { productivo rural de la } \\
\text { provincia E }\end{array}$ & $\begin{array}{l}\text { Reducción de } \\
\text { enfermedades } \\
\text { respiratorias y TBC } \\
\text { en la población } \\
\text { especialmente en } \\
\text { niños. } \\
\text { Seguridad } \\
\text { alimentaría }\end{array}$ & $\begin{array}{l}\text { Línea de base ex } \\
\text { ante y ex post del } \\
\text { proyecto. } \\
\text { Inventario } \\
\text { anual físico } \\
\text { socioambiental } \\
\text { de la gran cuenca } \\
\text { hidrográfica del Rio } \\
\text { Llanero, provincia } \\
\text { E. }\end{array}$ & $\begin{array}{l}\text { Política } \\
\text { macroeconómica } \\
\text { del país se } \\
\text { mantiene estable } \\
\text { en el largo plazo. } \\
\text { La apertura de la } \\
\text { economía peruana } \\
\text { consolidada a } \\
\text { través del libre } \\
\text { comercio nacional } \\
\text { e internacional }\end{array}$ \\
\hline
\end{tabular}

Fuente: Proyectos ganadores de concursos Fondoempleo, 2006-2009.

En este caso, observamos que ninguno de los dos indicadores presentados reúne las condiciones para ser considerados como tal; ambos no toman en cuenta los estándares de cantidad, calidad y tiempo, fundamentales para cualquier indicador. Además, se encuentra que en ninguno de los ejemplos presentados se consideran aspectos de mejora laboral o fomento del empleo, tal como lo requieren los manuales de Fondoempleo.

\section{Conclusiones}

Como se ha observado, la importancia de la aplicación del enfoque de marco lógico en los proyectos sociales de desarrollo es reconocida dada su simplicidad de implementación, sin embargo, también se encontraron dificultades en cuanto que, por ese mismo beneficio, no permite incluir toda la complejidad de las problemáticas sociales reales, además de considerarse una herramienta rígida. 
Encontramos que no hay una política pública que establezca criterios claros y comunes para el uso del marco lógico de manera adecuada y rigurosa en todos los niveles de gobierno (nacional, regional y local). Cada dependencia estatal, tiene su propia metodología, con variantes la mayoría de las veces contradictorias entre sí y orientados al diseño de proyectos con objetivos e indicadores inconsistentes que no tienen ninguna posibilidad de ser logrados o alcanzados.

Dada esta situación, constantemente los diseñadores de proyectos tienen que sujetarse a las propuestas metodológicas de cada fuente financiera internacional o nacional; las cuales, en su mayor parte exigen que los proyectos se comprometan a contribuir con la reducción de la pobreza o al logro de algún otro objetivo que, por su envergadura, es imposible de alcanzar desde un simple proyecto o programa de desarrollo.

Por todas estas inconsistencias, es que el problema central del uso del enfoque de marco lógico se vincula a su aplicación inadecuada. Así, como se observó previamente, la finalidad, sus objetivos e indicadores suelen ser inmedibles o, simplemente, no se incluyen. Asimismo, en la mayoría de los proyectos, no hay una adecuada relación de causa/efecto entre el propósito y la finalidad del proyecto. Otro aspecto importante lo constituyen los indicadores verificables objetivamente de los proyectos, ya que se encuentra que estos están referidos a aspectos genéricos y/o sin posibilidad de medición, en términos de cantidad, calidad y tiempo.

Consideramos que, superadas estas inconsistencias y con un uso adecuado del enfoque de marco lógico, se pueden diseñar proyectos que, gestionados adecuadamente y con eficiencia y eficacia, realmente podrán contribuir de manera sostenible a superar un problema específico, enmarcado en las políticas y planes de desarrollo de una localidad, región y del país. 


\section{Bibliografía}

Alcázar, L. (2007). ¿Por qué no funcionan los programas alimentarios y nutricionales en el Perú? Riesgos y oportunidades para su reforma. En Grupo de Análisis para el Desarrollo, Investigación, políticas y desarrollo en el Perú (p. 185-234). Lima: GRADE.

Baca, N. y Herrera, F. (2016). Proyectos sociales. Notas sobre su diseño y gestión en territorios rurales. Convergencia. Revista de Ciencias Sociales, 23(72), 69-87. Banco Mundial. (2004). Seguimiento/Evaluación: Instrumentos, métodos y enfoques. Banco Mundial.

Banco Interamericano de Desarrollo. (2004). El Marco Lógico para el Diseño de Proyectos. Banco Interamericano de Desarrollo.

Banco Mundial. (s.f). Matriz de Marco Lógico. Una herramienta de formulación de proyectos. Obtenido de http://www.disaster-info.net/lideres/spanish/mexico2004/biblio/ Marco.pdf

Bueno, C. (2014). Metodología del Marco Lógico en el proyecto de conformidad de servicios de proyectos sociales en la ONG Serpar. (Tesis para Licenciatura). Universidad Nacional del Centro.

Cohen, E. y Franco, R. (1988). Evaluación de proyectos sociales. Buenos Aires: Grupo Editor Latinoamericano.

Cohen, E. y Martínez, R. (2004). Formulación, evaluación y monitoreo de proyectos sociales. Chile: CEPAL.

Comisión Europea. (2002). Guía: Gestión del Ciclo de Proyectos. Segunda versión. Comisión Europea, PARTICIP GmbH.

Culebro, J., y Barragán, O. (2014). La Metodología del Marco Lógico en la administración pública federal. Nuevas formas de regulación para el control estratégico. Espacios públicos, 14(40), 33-48.

Fondo Internacional para el Desarrollo Agropecuario (2002). Gestión orientada al Impacto en el Desarrollo Rural. Guía para el S/E de proyecto. Fondo Internacional para el Desarrollo Agropecuario.

Franco, R. (1996). Los paradigmas de la política social en América Latina. CEPAL.

Gonzales, M. y Zanfrillo, A. (2006). Evaluación de proyectos en las Instituciones de Educación Superior: su abordaje a través del Marco Lógico. Técnica Administrativa, 5(28).

González-Anleo, J. (1999). Perspectiva sociológica de la organización. Cuadernos de estrategia, 4, 29-46.

GTZ. Agencia de Cooperación de Alemania. (1997). Planificación de Proyectos Orientada a Objetivos (ZOPP). GTZ.

Izuzquiza, I. (2008). La sociedad sin hombres: Niklas Luhmann o la teoría como escándalo. Barcelona: Anthropos. 
Loor, P., Loor, L. y Bravo, D. (2012). El Sistema de Marco Lógico (SML) y la necesidad de aplicación en proyectos de vinculación con la sociedad. ECA Sinergia, 3(1), 36-41.

Mejía, J. (2004). Sobre la investigación cualitativa. Nuevos conceptos y campos de desarrollo. Investigaciones sociales, 8(13), p. 277-299.

Ministerio de Economía y Finanzas. (2003). Guía General de Identificación, Formulación y Evaluación Social de Proyectos de Inversión Pública a nivel de Perfil. Lima: Dirección General de Programación Multianual del Sector Público.

Perrow, C. (1993). Una sociedad de organizaciones. Reis, 59, 19-55.

Powell, W. y Dimaggio, P. (1999). El nuevo institucionalismo en el análisis organizacional. México D.F.: Fondo de Cultura Económica.

Rodríguez, C. (2008). Sistema de información basados en indicadores de seguimiento de proyectos en organizaciones de cooperación descentralizada. Estudio de caso de los proyectos cofinanciados por la Comunidad de Madrid en Perú. (Tesis para Doctorado). Universidad Politécnica de Madrid.

Saavedra, J. y Chacaltana, J. (2000). El programa de capacitación laboral juvenil (Projoven). Boletín Cinterfor, 150, 151-162.

Sánchez, N. (2007). El marco lógico. Metodología para la planificación, seguimiento y evaluación de proyectos. Visión Gerencial, 2, 328-343.

Sánchez, M. (2011). El uso de la metodología de Marco Lógico en el diseño y la evaluación: alcances y limitaciones. El programa Hábitat y el Plan Municipal de Desarrollo, Puebla 2008-2011. (Tesis para Maestría). Flacso. 